\title{
Steric pressure among membrane-bound polymers opposes lipid phase separation
}

\author{
Zachary I. Imam ${ }^{1}$, Laura E. Kenyon ${ }^{1}$, Adelita Carrillo ${ }^{1}$, Isai Espinoza ${ }^{1}$, Fatema Nagib ${ }^{1}$, and \\ Jeanne C. Stachowiak ${ }^{1,2,{ }^{*}}$ \\ ${ }^{1}$ Department of Biomedical Engineering, The University of Texas at Austin, TX \\ ${ }^{2}$ Institute for Cellular and Molecular Biology, The University of Texas at Austin, TX
}

\begin{abstract}
Lipid rafts are thought to be key organizers of membrane-protein complexes in cells. Many proteins that interact with rafts have bulky polymeric components such as intrinsically disordered protein domains and polysaccharide chains. Therefore, understanding the interaction between membrane domains and membrane-bound polymers provides insights into the roles rafts play in cells. Multiple studies have demonstrated that high concentrations of membrane-bound polymeric domains create significant lateral steric pressure at membrane surfaces. Furthermore our recent work has shown that lateral steric pressure at membrane surfaces opposes the assembly of membrane domains. Building on these findings, here we report that membrane-bound polymers are potent suppressors of membrane phase separation, which can destabilize lipid domains with substantially greater efficiency than globular domains such as membrane-bound proteins. Specifically, we created giant vesicles with a ternary lipid composition, which separated into coexisting liquid ordered and disordered phases. Lipids with saturated tails and polyethylene glycol (PEG) chains conjugated to their head groups were included at increasing molar concentrations. When these lipids were sparse on the membrane surface they partitioned to the liquid ordered phase. However, as they became more concentrated, the fraction of GUVs that were phase-separated decreased dramatically, ultimately yielding a population of homogenous membrane vesicles. Experiments and physical modeling using compositions of increasing PEG molecular weight and lipid miscibility phase transition temperature demonstrate that longer polymers are the most efficient suppressors of domain phase separation when the energetic barrier to lipid mixing is low. In contrast, as the miscibility transition temperature increases, longer polymers are more readily driven out of domains by the increased steric pressure. Therefore, the concentration of shorter polymers required to suppress phase separation decreases relative to longer polymers. Collectively, our results demonstrate that crowded, membrane-bound polymers are highly efficient suppressors of phase separation and suggest that the ability of lipid domains to resist steric pressure depends on both their lipid composition and the size and concentration of the membrane-bound polymers they incorporate.
\end{abstract}

\section{Graphical abstract}

*To whom correspondence should be addressed: Jeanne Stachowiak (jcstach@austin.utexas.edu). SUPPLEMENTARY INFORMATION

Supplementary figures are included with this manuscript. 


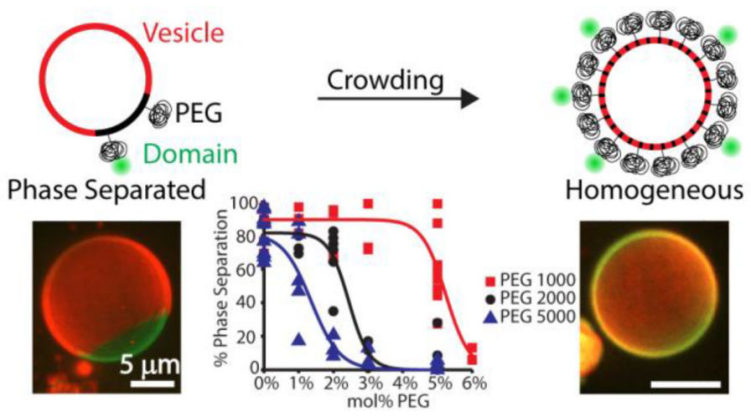

\section{INTRODUCTION}

Cellular membranes are populated by diverse transmembrane and membrane-bound proteins, which must be spatially and temporally organized to facilitate assembly of cellular structures including signaling complexes, ${ }^{1,2}$ trafficking vesicles, ${ }^{3,4}$ and cytoskeletal protrusions. ${ }^{5}$ Among the key mechanisms thought to drive membrane organization is the concentration of specific protein and lipid species by membrane rafts. ${ }^{6}$ Membrane rafts are hypothesized to be dynamic, nanoscale, membrane structures which are enriched in sphingolipids, cholesterol and other protein and lipid constituents. ${ }^{6}$ Numerous studies have explored the phase behavior of lipids ${ }^{7-10}$ and the preferences of specific proteins for liquid ordered and liquid disordered environments, ${ }^{11-13}$ suggesting that membrane rafts help to sort proteins at cellular surfaces.

As proteins become concentrated in rafts, protein-protein interactions are expected to increase, driving assembly of caveolae, ${ }^{14}$ viral buds, ${ }^{15,16}$ and signaling complexes. ${ }^{17,18}$ However, non-specific steric interactions among proteins will also increase as proteins become crowded in the raft environment. Paradoxically, our recent work showed that steric interactions among membrane-bound proteins can create pressure that opposes the separation of membranes into liquid ordered $\left(\mathrm{L}_{\mathrm{O}}\right)$ and liquid disordered $\left(\mathrm{L}_{\mathrm{D}}\right)$ phases, destabilizing liquid ordered regions known as lipid domains ${ }^{19}$. In line with the idea that steric pressure suppresses membrane phase separation, the concentration of membranebound proteins required to drive the process decreased as protein size increased. These findings are important because they were the first to reveal the substantial entropic cost of concentrating biomolecules within lipid domains, highlighting the delicate energetic balance that underlies domain assembly and stability.

In line with this thinking, some of the largest molecules at membrane surfaces are disordered polymeric domains. For example, more than $40 \%$ of transmembrane proteins have substantial intrinsically disordered domains, ${ }^{20}$ which occupy significantly greater volume than ordered domains of equivalent molecular weight. ${ }^{21}$ Transmembrane proteins that contain these disordered domains are diverse, comprising $\mathrm{G}$ protein coupled receptors, immunological receptors, cell adhesion proteins, ion channels, and others. ${ }^{22}$ Similarly, polysaccharide chains associated with glycolipids and glycosylated proteins occupy a considerable fraction of cellular membrane surfaces, ${ }^{23}$ and are frequently associated with lipid rafts. ${ }^{16,24,25}$ Membrane-bound polysaccharide chains form a layer known as the 
glycocalyx that is commonly $50-100 \mathrm{~nm}$ thick, ${ }^{26}$ an order of magnitude thicker than the layer of transmembrane proteins at the membrane surface. ${ }^{27}$

In addition to their prevalence at cellular membrane surfaces, polymeric domains typically occupy significantly greater volumes than well-folded biomolecules of equivalent molecular weight. Specifically, polymer radii increase with increasing molecular weight to approximately the one-half power $\left(\mathrm{R} \sim \mathrm{M}_{\mathrm{w}}^{1 / 2}\right)$, while the radii of globular proteins increase with molecular weight to approximately the one-third power $\left(R \sim M_{w}^{1 / 3}\right){ }^{28}$ The large size of polymeric domains suggests their potential to crowd membrane surfaces, producing steric pressure. Several studies have shown that membrane-conjugated polymers such as polyethylene glycol (PEG) chains can generate significant lateral steric pressure, sufficient to influence lipid phase behavior ${ }^{29}$ and at very high densities, sufficient to induce formation of coexisting gel-gel phases owing to depletion attraction effects. ${ }^{30-32}$ Additionally, simply attaching polymers to the membrane surface comes at an entropic $\cos ^{33}$ such that polymers prefer curved surfaces ${ }^{34}$ and sterically repel other molecules including other PEG chains. ${ }^{35}$ This ability of PEG-conjugated lipid membranes to sterically exclude other molecules can prevent non-specific biochemical interactions, ${ }^{36}$ a property which has made PEG-conjugated membranes popular as delivery vehicles in pharmaceutical applications, ${ }^{36}$ and as inert substrates and barriers for biophysical experiments. ${ }^{37,38}$ For example, polymer layers have been used as a cushion to suspend bilayers above substrates. ${ }^{39}$ Previous studies on PEGconjugated monolayers have also revealed evidence of steric pressure influencing membrane organization. ${ }^{40}$ Taken together, previous work clearly demonstrates that membrane-bound polymers such as PEG chains strongly resist lateral compression, enabling them to crowd membrane surfaces.

Recognizing the large size and ubiquitous presence of polymeric domains at membrane surfaces, here we investigate the impact of steric pressure generated by membrane-bound polymers on the process of membrane phase separation. Specifically, we examined the impact of increasing concentrations of PEG-conjugated lipids on the stability of micronscale liquid-liquid phase co-existence in giant unilamellar vesicles (GUVs). Our results reveal that the large projected area of PEG chains at the membrane surface in comparison globular molecules of similar molecular weight ${ }^{19}$ makes them highly efficient suppressors of membrane phase separation. As such, the longest PEG chains were the most efficient in terms of the molar concentration required to destabilize membrane phase separation. However as the energetic barrier to lipid mixing increased, the increased steric pressure required to destabilize lipid domains began to compete with the tail-driven preference of PEG-conjugated lipids for the ordered phase. Reduced partitioning of PEG-conjugated lipids to the ordered phase diminished the ability of the PEG chains to generate steric pressure, an effect that disproportionately impacted longer PEG chains, reducing their advantage over shorter chains. These findings provide insight into the competition that membrane-bound macromolecules experience between preference for the lipid domain environment and rising steric pressure as they accumulate in lipid domains. Collectively, this work demonstrates that membrane-bound polymers can have a major impact on the stability of lipid domains, suggesting that polymeric biomolecules may be major determinants of the content and stability of lipid rafts. 


\section{RESULTS AND DISCUSSION}

We began by investigating the impact of PEG-conjugated lipids on the phase behavior of a ternary system consisting of 45 mol\% DOPC (1, 2-dioleoyl-sn-glycero-3-phosphocholine), 25 mol\% DPPC (1, 2-dipalmitoyl-sn-glycero-3-phosphocholine), and $30 \mathrm{~mol} \%$ Cholesterol, which we will refer to as lipid composition 1 (Figure 1A). The lipid probe Texas Red-DPPE (Texas Red 1, 2-dipalmitoyl-sn-glycero-phosphoethanolamine), which is known to partition into the majority liquid disordered phase ${ }^{41,42}$ was included at $0.3 \mathrm{~mol} \%$ to visualize the phases (Figure 1B). The miscibility phase transition temperature of the chosen lipid composition in the absence of PEG-conjugated lipids is approximately $29^{\circ} \mathrm{C}, 4^{\circ} \mathrm{C}$ above the observation temperature of $25^{\circ} \mathrm{C}$. $^{7}$ Therefore, as expected, in the absence of PEGconjugated lipids a substantial majority of vesicles, $82 \%$, displayed distinct phase separation. In phase separated vesicles the majority of the vesicle surface appeared bright in the Texas Red fluorescence channel, with darker, rounded micron-scale ordered phase domains present on the vesicle surface. These domains occupied on average $22 \%$ of the vesicle surface based on confocal analysis of domain size (see Methods), in approximate agreement with the literature ${ }^{43}$ Next we repeated these experiments with giant vesicles containing 1, 2, 3, and 5 mol\% PEG2000-DPPE (1, 2-dipalmitoyl-sn-glycero-3-phosphoethanolamine-N-

[methoxy(polyethylene glycol)-2000]), a lipid with two fully saturated tails and a PEG chain of 2000 Da molecular weight conjugated to the head group (Figure 1C). In each case the concentration of DPPC was reduced accordingly to accommodate the PEG-conjugated lipids while maintaining the same total fraction of lipids with saturated tail groups (ie. $25 \mathrm{~mol} \%$ ).

As the concentration of PEG2000-DPPE increased from 0 to $5 \mathrm{~mol} \%$, the fraction of phase separated vesicles dropped monotonically from $82 \% \pm 1.3$ (standard error of mean) in the absence of PEG to $12 \% \pm 4.8$ for vesicles containing 5 mol\% PEG2000-DPPE, with the steepest portion of the transition occurring between 2 and 3 mol\% PEG2000-DPPE (Figure 1D). Phase separated vesicles were replaced by an increasing proportion of vesicles that displayed a homogenous distribution of both Texas Red DPPE and a Carboxyfluoresceinlabeled version of the PEG-conjugated lipid (fPEG2000-DSPE), suggesting that inclusion of PEG prevented the formation of ordered domains. However, we cannot rule out the possibility that phase separated regions with sub diffraction limited dimensions were still present on the membrane surface. The correlation of the suppression of membrane phase separation with increasing concentration of PEG-conjugated lipids, $\mathrm{C}_{\mathrm{PEG}}$, as well as our previous work showing that steric pressure among membrane-bound proteins can suppress $\mathrm{L}_{\mathrm{O}^{-}} \mathrm{L}_{\mathrm{D}}$ phase separation ${ }^{19}$ suggest that steric interactions among PEG chains are opposing membrane phase separation in these experiments.

If steric interactions among membrane-bound PEG chains suppress membrane phase separation, then as the molecular weight of the PEG chain increases, the number of PEG chains per membrane area required to suppress phase separation should decrease. To test this hypothesis we repeated our GUV experiments using lipids conjugated with $1 \mathrm{kDa}$ and $5 \mathrm{kDa}$ molecular weight PEG chains, PEG1000-DPPE and PEG5000-DPPE, respectively. For each PEG chain length, the fraction of GUVs that displayed micron-scale liquid-liquid immiscibility decreased from more than $80 \%$ in the absence of PEG to less than $13 \%$ as $\mathrm{C}_{\mathrm{PEG}}$ increased, Figures $2 \mathrm{~A}-\mathrm{D}$. The value of $\mathrm{C}_{\mathrm{PEG}}$ required to drive this transition 
decreased with increasing PEG chain length. For example, at $\mathrm{C}_{\mathrm{PEG}}$ equal to $2 \mathrm{~mol} \%$, the majority of vesicles containing PEG1000-DPPE (Figure 2A) and PEG2000-DPPE (Figure 2B) were phase separated, while the majority of vesicles containing PEG5000-DPPE had become homogenous (Figure 2C). The steepest portion of the transition occurred at 1-2 mol $\%$ for PEG5000-DPPE, 2-3 mol \% for PEG2000-DPPE (as reported in Figure 1, repeated for visual comparison), and 5-6 mol\% for PEG1000-DPPE (Figure 2D). Since larger PEG chains cover a larger fraction of the membrane surface, this trend is qualitatively consistent with the hypothesis that steric pressure among PEG chains suppresses phase separation (Figure 2E). Interestingly, near these transition points, the ordered domain broke up into many small ordered domains (Figure S1), similar to our previous observations of domain breakup driven by protein crowding. ${ }^{19}$

To further evaluate the potential of steric pressure to suppress membrane phase separation we constructed a simplified analytical model of steric interactions among crowded, membrane-tethered PEG chains and compared the resulting surface pressure to estimates of the enthalpy of lipid mixing. We chose the two dimensional form of the Carnahan-Starling equation of state ${ }^{44,45}$ to estimate the pressure generated by crowded PEG chains. This equation approximates the PEG chains as self-avoiding, non-deformable spherical particles with radii equal to the polymer radii of gyration, $R_{g}$, where estimates of $R_{g}$ were made using a freely-joined chain model and found comparable to experimental values taken from the literature: (1.0 nm for PEG1000, $1.5 \mathrm{~nm}$ for PEG2000, $2.3 \mathrm{~nm}$ for PEG5000). ${ }^{32}$ The Carnahan-Starling equation predicts that the pressure $(p)$ generated by crowding at the membrane surface, with units of energy per membrane area, should increase nonlinearly as the fractional polymer coverage of the membrane surface $\left(\eta\left(A_{P E G}\right)\right.$ increases,

$$
p=\frac{\eta\left(A_{P E G}\right)}{A_{P E G}}\left\{1+2 \eta\left(A_{P E G}\right) \frac{1-0.44 \eta\left(A_{P E G}\right)}{\left[1-\eta\left(A_{P E G}\right)\right]^{2}}\right\} k_{B} T
$$

(Equation 1)

where $A_{P E G}$ is the area occupied by individual PEG molecules on the membrane surface, $k_{B}$ is Boltzmann's constant, and $T$ is absolute temperature. Here longer PEG chains occupy larger areas on the membrane surface, such that $\eta$ is a linear function of $A_{P E G}$.

In Figure 3A the pressure generated by the polymers is plotted as a function of the molar concentration of PEG conjugated lipids. The horizontal line is the approximate enthalpy of mixing for a typical phase-separated lipid mixture, $4.8 \frac{\mathrm{mJ}}{\mathrm{m}^{2}} .46$ Therefore the concentration of PEG-conjugated lipids required to suppress membrane phase separation can be estimated from the intersection between the enthalpy of mixing and the pressure generated by the membrane-bound polymer. Notably the enthalpy of mixing value used in this study represents a first order estimate. However, the comparison between the model and the data is not highly sensitive to the value of the enthalpy of mixing owing to the steep increase in the steric pressure as the coverage of PEG chains on the membrane surface becomes substantial (Figure 3A). 
To assess the agreement between the simple steric pressure model and the experimental results, the observed transition points were plotted versus the predicted transition points (Figure 3B). The experimentally observed transition points were estimated to be $1.4 \mathrm{~mol} \%$, $2.4 \mathrm{~mol} \%$, and $5.2 \mathrm{~mol} \%$ for PEG5000-DPPE, PEG2000-DPPE, and PEG1000-DPPE, respectively, within the ordered membrane phase (see Methods). The plot demonstrates reasonable agreement between theory and experiment, suggesting that the steric pressure generated by polymer-polymer interactions on the membrane surface is sufficient to suppress membrane phase separation as $\mathrm{C}_{\mathrm{PEG}}$ is increased (Figure 3B).

Approximating the flexible membrane-conjugated PEG chains as rigid spherical particles assumes that the lateral steric pressure at the membrane surface is insufficient to significantly straighten the PEG chains. This assumption is reasonable because the membrane area per PEG molecule at the mixing transition for each of the PEG chain lengths $\left(6.8 \mathrm{~nm}^{2} / \mathrm{PEG}\right.$ for PEG1000, $11 \mathrm{~nm}^{2} / \mathrm{PEG}$ for PEG2000, $23 \mathrm{~nm}^{2} / \mathrm{PEG}$ for PEG5000) are all significantly larger than the projected area of the molecules $\left(3.4 \mathrm{~nm}^{2}\right.$ for PEG1000, $6.7 \mathrm{~nm}^{2}$ for PEG2000, $17 \mathrm{~nm}^{2}$ for PEG5000), such that the molecules are not substantially compressed at the concentrations used in this work. Further, the energetic cost of fully straightening a polymer chain can be approximated as $\Delta G_{\text {straight }} \approx N k_{B} T$, where $k_{B}$ is the Boltzmann's constant, $T$ is temperature and $N$ is the number of PEG monomers (23 monomers for $1 \mathrm{kDa}, 46$ for $2 \mathrm{kDa}, 114$ for $5 \mathrm{kDa}$ ). Dividing this free energy by the area per molecule, which is also proportional to $N$, gives the energetic cost of straightening per membrane area, approximately $28 \frac{\mathrm{mJ}}{\mathrm{m}^{2}}$, substantially larger than the energy per area required to suppress membrane phase separation, $4.8 \frac{\mathrm{mJ}}{\mathrm{m}^{2}}$.

The data and theoretical arguments presented so far support the hypothesis that steric pressure among membrane-tethered polymers potently suppresses membrane phase separation. However, from these data alone it is not clear how the process will change when the energetic barrier to lipid mixing increases. In particular, how will the lipid mixing process be different when steric pressure within the ordered lipid phase begins to compete with the finite preference of PEG-conjugated lipids for this phase? To examine these questions we analyzed the partitioning of labeled PEG2000-conjugated lipids ( $0.1 \mathrm{~mol} \%)$ to the ordered phase as a function of increasing PEG2000-DPPE concentration. Here partition factor is defined as the ratio of the fluorescence intensity (background subtracted) of the labeled PEG-conjugated lipid in the ordered and disordered phases (Figure 4A). Plotting these data together with the fraction of phase separated vesicles reveals that the transition to a majority of homogenous vesicles, which occurs between 2 and 3 mol\% PEG-conjugated lipids, is slightly preceded by a significant decline in the partition factor of labeled, PEGconjugated lipids to the ordered phase (Figure 4B). These data suggest that the steric pressure that must be generated by the PEG molecules to suppress membrane phase separation also competes with their own energetic preference for the ordered phase. We would expect this competition to become more significant as the energetic barrier to lipid mixing increases, since a greater steric pressure will be required to overcome the increased barrier. To examine this prediction we increased the fraction of saturated lipids (DPPC) in the membrane, forming vesicles with composition 2 (Figure 4C): $34 \mathrm{~mol} \%$ DPPC, $33 \mathrm{~mol} \%$ 
Cholesterol, and $33 \mathrm{~mol} \%$ DOPC. For vesicles of composition 2, plotting fractional phase separation and the partition factor of labeled PEG lipids to the ordered phase as a function of $\mathrm{C}_{\mathrm{PEG}}$ shows that while the partition factor still drops significantly between 0.1 and $2 \mathrm{~mol} \%$ $\mathrm{C}_{\mathrm{PEG}}$ and stabilizes after $2 \mathrm{~mol} \% \mathrm{C}_{\mathrm{PEG}}$, the major reduction in the fraction of phase separated vesicles requires a significantly higher value of $\mathrm{C}_{\mathrm{PEG}}$ than it did for vesicles of composition 1, between 5 and $10 \mathrm{~mol} \%$ (Figure 4D).

As further evidence that repartitioning of labeled PEG lipids to the disordered phase precedes lipid mixing, Figure 5 shows line profiles of florescence intensity for Texas RedDPPE, a marker of the disordered phase, and fPEG2000-DSPE. From these images and profiles it is clear that while the labeled, PEG-conjugated lipid is strongly partitioned to the ordered phase for vesicles containing 1 mol\% PEG2000-DPPE (Figure 5A, B), the extent of partitioning declines significantly for vesicles containing 5 mol\% PEG2000-DPPE (Figure 5C, D). In contrast, Texas Red-DPPE partitions strongly to the disordered phase for both molar fractions of PEG2000-DPPE, indicating that the vesicles are still phase separated. Notably, since DSPE has a higher melting temperature than DPPE, the fPEG2000-DSPE lipid is likely to partition somewhat more strongly to the ordered phase in comparison to the PEG2000-DPPE lipid. Therefore, we expect that PEG2000-DPPE begins to repartition significantly to the liquid disordered phase at slightly lower molar concentrations than those predicted by the repartitioning of the fPEG2000-DSPE probe lipid in Figure 4D.

These results confirm that steric pressure among membrane-bound PEG chains competes significantly with their tail-based preference for the ordered phase. This effect should lead to diminishing returns in the ability of PEG chains to concentrate in the ordered phase as the concentration of PEG-conjugated lipids increases. Specifically, as $\mathrm{T}_{\mathrm{m}}$ increases, the steric pressure required to suppress phase separation must increase. This requirement for increased steric pressure will in turn dictate an increase in the concentration of PEG-conjugated lipids required to destabilize membrane phase separation, for two reasons. First, even in the limit of perfect partitioning of PEG-conjugated lipids to the ordered phase, increasing the steric pressure requires increasing the concentration of PEG-conjugated lipids. Second, based on the data in Figures 4 and 5, the increase in steric pressure is expected to reduce the partitioning of PEG-conjugated lipids to the ordered phase, such that for each incremental increase in the overall concentration of PEG-conjugated lipids, the concentration in the ordered phase should increase by a smaller and smaller amount. As a result of these phenomena, an increasing total concentration of PEG-conjugated lipids was needed to destabilize membrane phase separation as $T_{m}$ increased (compare Figures 4 B, D).

We would expect the significance of this effect to increase with increasing PEG chain length for at least two reasons. First, the slope of the pressure-concentration relationship in Figure $3 \mathrm{~A}$, which has units of energy per mole of PEG, can be interpreted as the energetic cost of an incremental increase in the number of PEG-conjugated lipids within the ordered phase, when domain area is held constant. The increase in this slope with increasing PEG chain length at constant steric pressure indicates that the thermodynamic work that must be done to accommodate an additional PEG-conjugated lipid within the crowded population of membrane-bound PEG chains increases with increasing PEG size. Second, at a given concentration of PEG-conjugated lipids, longer PEG chains cover a greater fraction of the 
membrane surface and are therefore more likely to reach concentrations at which steric interactions with neighboring PEG chains will drive them to straighten. In our discussion of composition 1 we neglected chain straightening, since the membrane coverage within the ordered domain remained well below $100 \%$ coverage, based on the projected area of the PEG chains. However, as PEG concentration increases, the longest PEG chains, which occupy the most membrane surface area, will begin to reach $100 \%$ coverage. For example PEG5000-DPPE lipids are expected to cover $100 \%$ of the ordered domain surface when domains contain $5 \mathrm{~mol} \%$ or more of the lipid. As the concentration of the lipid approaches and exceeds this threshold, significant straightening of the PEG chains will begin. Chain straightening will increase the energetic cost of retaining PEG-conjugated lipids in the domain both by increasing the slope of the pressure-concentration curve and by decreasing the conformational entropy of the PEG chains within the domain. ${ }^{47}$ The sum of these energetic costs, all of which increase with increasing PEG chain length, must be balanced by the tail-based energetic preference of the lipid for the ordered phase (Figure 7A). Since this preference should not depend on PEG chain length, we expect PEG-conjugated lipids to partition less and less favorably to the ordered phase as PEG chain length increases.

Figure 6 plots the fraction of phase-separated vesicles of composition 2 as a function of increasing concentration of PEG-conjugated lipids of all lengths: PEG1000-DPPE, PEG2000-DPPE (repeated from Fig. 4 for visual comparison), and PEG5000-DPPE. As anticipated, increasing the energetic barrier increases the molar concentration of PEG required to suppress membrane phase separation. In particular, for vesicles containing $5 \mathrm{~mol}$ $\%$ of PEG-conjugated lipids of all chain lengths, the majority of vesicles of composition 2 remain phase separated (Figure $6 \mathrm{~A}-\mathrm{C}$ ), whereas vesicles of composition 1 containing $5 \mathrm{~mol}$ $\%$ PEG2000-DPPE and PEG5000-DPPE were homogenous (Figure 2D). For vesicles of composition 2 , the transition from a majority of phase-separated vesicles to a majority of homogenous vesicles occurs when $\mathrm{C}_{\mathrm{PEG}}$ is between 5-10 mol\% for all PEG chain lengths (Figure 6D). While this concentration range is similar to the molar fraction of the smallest PEG-conjugated lipid (PEG1000-DPPE) required to suppress membrane phase separation for composition 1, the fraction of the largest PEG-conjugated lipid (PEG5000-DPPE) shifted substantially from just $1-2 \mathrm{~mol} \%$ for composition 1 to $5-10 \mathrm{~mol} \%$ for composition 2 . As reported above, the transition concentration for the intermediate sized PEG-conjugated lipid (PEG2000-DHPE) shifted by an intermediate amount, requiring 2-3 mol\% for composition 1 (Figure 2D) and 5-10 mol\% for composition 2. Additionally, vesicles of composition 2 exhibited broken up ordered domains between 2 and 5 mol\% PEG-DPPE similar to what was observed near the transition points in composition 1, Figure S2.

These results illustrate the expected diminishing returns in the capacity of crowded, membrane-bound PEG chains to destabilize lipid domains as PEG chain length and the energetic barrier to lipid mixing both increase. To demonstrate this point, Figure 6E, F plot the approximate molar concentrations of PEG-conjugated lipids required to suppress membrane phase separation for each of the PEG chain lengths for compositions 1 and 2. For composition 1, as PEG chain length increases, the concentration of PEG-conjugated lipids required to suppress phase separation decreases dramatically. In comparison, for composition 2 this decrease is substantially smaller. If we consider instead the ratio of total PEG mass to total lipid mass, we can determine the efficiency with which a polymer 
opposes membrane phase separation. Specifically, a polymer is judged to be more efficient at suppressing phase separation if a smaller total mass of polymer molecules per mass of lipid molecules is required to disrupt lipid domains. For composition 1 there is a slight increase in this ratio with increasing PEG chain length (Figure 6G). In contrast, for composition 2, the increase in the ratio with increasing PEG chain length is significantly larger. Specifically, as PEG chain length decreases, the mass ratio of PEG chains to lipids required to suppress phase separation decreases demonstrating the superior efficiency of shorter PEG chains at destabilizing lipid domains as the energetic barrier to lipid mixing increases (Figure 6H). However, regardless of PEG chain length, the ratio of PEG to lipid mass required to suppress membrane phase separation is more than an order of magnitude smaller than the ratio of globular protein to lipid mass required to destabilize lipid domains in our previous work (data not shown in this document). ${ }^{19}$ This finding illustrates the potent capacity of extended, membrane-bound polymers to influence lipid phase behavior by generating steric pressure at membrane surfaces.

\section{CONCLUSIONS}

Here we have demonstrated that steric pressure among membrane-bound polymers potently suppresses membrane phase separation. Given the high concentration of glycosylated and intrinsically disordered proteins at membrane surfaces ${ }^{20,26}$ membrane-bound polymers are likely to be among the key determiners of steric pressure and lipid domain stability at cellular surfaces. One possible biological implication of our findings is that proteins and lipids with large polymeric conjugations may be difficult to confine in lipid rafts owing to the steric pressure they create at the crowded cellular surface. For example, proteoglycans such as syndecan-4, which have large heparin sulfate conjugations, have been found to enter lipid rafts only when driven to oligomerize by the presence of external binding partners such as growth factors and antibodies. ${ }^{48}$ Here the ability of binding interactions to change the membrane phase preference of the protein is consistent with the idea of an external energy input being necessary to concentrate bulky polymeric domains within the confined environment of a phase separated lipid domain.

Further, expanding upon our earlier work, results in this paper reveal that crowding molecule size, concentration, affinity for lipid domains, and domain stability all come together to determine which membrane-bound polymers are the most effective suppressors of membrane phase separation (Figure 7). Specifically, when the energetic barrier to lipid mixing is relatively low, the concentration of PEG-conjugated lipids required to destabilize domains increases with decreasing polymer projected area on the membrane surface (Figure 7B), in agreement with a simple analytical model of steric pressure among membrane-bound particles (Figure 3A), which assumes that all PEG-conjugated lipids partition equally to the ordered phase. In contrast, as the energetic barrier to lipid mixing is increased, the steric pressure required for lipid mixing increases and PEG-conjugated lipids begin to partition significantly less favorably to the ordered phase (Figure 7C,D). This effect diminishes the advantage of long PEG chains, favoring shorter chains that pay a smaller energetic cost to remain in the ordered phase at a given pressure. Therefore, as pressure increases and membrane-bound polymers begin to repartition outside the domain, smaller polymers are increasingly efficient at overcoming larger energetic barriers to lipid mixing. As such, in the 
limit of very strong partitioning of polymers to the liquid-ordered domain, as would be expected for those conjugated to lipids with long saturated tails or multiple lipid anchors, large polymers are likely to be the most efficient at suppressing membrane phase separation. In contrast when steric pressure is high enough to significantly reduce partitioning of membrane-bound polymers to the ordered phase, smaller polymers will partition to the ordered phase more strongly than larger polymers, enabling smaller polymers to destabilize lipid domains at a lower polymer to lipid mass ratio. Collectively, these results illustrate that membrane phase separation depends on a delicate energetic balance that can be manipulated by changes in steric pressure among crowded macromolecules at membrane surfaces.

\section{MATERIALS AND METHODS}

\section{Chemical reagents}

HEPES (4-(2-hydroxyethyl)-1-piperazineethanesulfonic acid was purchased from Sigma Aldrich. $\mathrm{NaCl}$ and sucrose were purchased from Fisher Scientific. Texas Red-DPPE (Texas Red- 1,2-Dipalmitoyl-sn- Glycero-3-phosphoethanolamine) was purchased from Invitrogen. DPPC (1,2 - dipalmitoyl-sn-glycero-3-phosphocholine), DOPC (1,2-dioleoyl-sn-glycero-3phosphocholine), cholesterol (from ovine wool), PEG1000-DPPE (1,2-Dipalmitoyl-snglycerol-3-phosphoethanolamine-N-[Methoxy(Polyethylene glycol)-1000]), PEG2000DPPE (1,2-Dipalmitoyl-sn-glycerol-3-phosphoethanolamine-N-[Methoxy(Polyethylene glycol)-2000]), PEG5000-DPPE (1,2-Dipalmitoyl-sn-glycerol-3-phosphoethanolamine-N [Methoxy(Polyethylene glycol)-5000]), and Carboxyfluorescein-PEG2000-DSPE (1,2 distearoyl-sn-glycero-3-phophoethanolamine-N-[poly(ethylene glycol) 2000-N'carboxyfluorescein]) were all purchased from Avanti Polar Lipids (Alabaster, AL). All reagents were used without further purification. Polymer molecular weight distribution did not exceed $\pm 25 \%$ of the indicated molecular weight according to the manufacturer of the PEG chains.

\section{Giant vesicles}

Giant Unilamellar Vesicle (GUV) electroformation was completed by following published protocols. ${ }^{49}$ The lipid compositions chosen were based on the well-studied DPPC:Chol:DOPC ternary lipid mixture. ${ }^{7}$ Composition 1 was $25 \mathrm{~mol} \%$ DPPC, $30 \mathrm{~mol} \%$ cholesterol, and 45 mol\% DOPC. The lipid mixtures with PEG-DPPE were created by adding 1, 2, 3, and 5 mol \% PEG-DPPE while removing 1, 2, 3, and 5 mol\% DPPC respectively, to maintain a constant total molar fraction of saturated lipids. Composition 2 was $34 \mathrm{~mol} \%$ DPPC, $33 \mathrm{~mol} \%$ cholesterol, and $33 \mathrm{~mol} \%$ DOPC. The lipid mixtures with PEG-DPPE were created by adding 1, 2, 5, 10, and 15 mol\% PEG-DPPE while removing 1 , $2,5,10$, and $15 \mathrm{~mol} \%$ DPPC respectively to maintain a constant total molar fraction of saturated lipids. The lipids were spread on inidium-tin-oxide (ITO) coated glass slides (resistance $~ 8-12 \Omega / \mathrm{sq}$ ) and placed under vacuum for at least 2 hours to remove all of the solvent. Electroformation was performed at approximately $55^{\circ} \mathrm{C}$ to exceed the highest expected melting temperature of DPPC, $41^{\circ} \mathrm{C} .{ }^{7}$ The vesicles were prepared using sucrose solution (320 milliosmole). In all experiments vesicle solution osmolarity was measured using a vapor pressure osmometer (Wescor). 


\section{GUV slide preparation}

After electroformation, the vesicles were diluted and prepared for imaging. Vesicles were diluted by a factor of 6 in $20 \mathrm{mM}$ HEPES buffer with $150 \mathrm{mM} \mathrm{NaCl}$, raised to a final osmotic strength of approximately $310 \mathrm{mOsm}$ by the addition of sucrose. The slight osmotic tension (vesicles at $320 \mathrm{mOsm}$ ) suppressed membrane shape fluctuations, which improved image quality and the reproducibility of results. Using this system, with sucrose solution inside the vesicles and salt-containing buffer outside could have a small effect on the phase behavior of the membrane ${ }^{50}$ Imaging experiments began approximately 5 minutes after the samples were prepared to allow the GUVs to settle to the bottom of the coverslip before imaging. In all cases the vesicles were observed in small sealed disposable chambers composed of glass cover slips and spacers made from double-sided tape.

\section{Microscopy}

Spinning disc confocal microscopy (Zeiss Axio Observer Z1 with Yokagawa CSU-X1M) was used to image the GUVs. Laser wavelengths of $488 \mathrm{~nm}$ and $561 \mathrm{~nm}$ were used for excitation. The bandpass emission filters were centered at $525 \mathrm{~nm}$ with a $50-\mathrm{nm}$ bandwidth, and $629 \mathrm{~nm}$ with a $62-\mathrm{nm}$ bandwidth. A Plan-Apochromat 100× 1.4 numerical aperture oil immersion objective was used for imaging. A cooled $\left(-70^{\circ} \mathrm{C}\right)$ EMCCD iXon3 897 camera was used for imaging (Andor Technology). The acquisition speed of each $\mathrm{z}$ stack experiment was 0.95 seconds per optical slice.

\section{Vesicle dimension measurements}

The average area of the liquid-ordered domains as a percentage of total vesicle surface area was estimated using confocal reconstructions of the domain. From these reconstructions the domain was considered to be a spherical cap for which the area was calculated based on measurements of the vesicle diameter and the diameter of the cap.

\section{Calculation of projected area per molecule of PEG chains on the membrane surface}

In order to determine the area that the PEG chain occupies on the membrane surface under dilute conditions, the radius of gyration was estimated using the following equation:

$R_{G}^{2}=\frac{N_{R L} a^{2}}{6}$ where $N_{R L}$ is the number of rigid lengths and a is the Kuhn length. The number of rigid lengths is determined by using the following equation: $N_{R L}=\frac{N_{\text {monomer }} l_{\text {monomer }}}{a}$ Where $N_{\text {monomer }}$ is the number of monomers, $I_{\text {monomer }}$ is the length of a monomer. Next the area of the PEG was calculated using the area equation $A_{P E G}=\pi R_{g}{ }^{2}$.

\section{Estimating the fraction of phase-separated vesicles in the GUV population}

Data were collected from three independent batches of GUVs. Ten image stacks per experimental condition were taken, each containing multiple vesicles. At least 30 GUVs per batch were counted per data point. The number of phase-separated GUVs and homogeneous GUVs were then counted. The phase separation percentage was determined by calculating the number of phase separated GUVs as a percentage of total GUVs. In order to be counted as a phase-separated GUV, the GUVs had to be at least $5 \mu \mathrm{m}$ in diameter, lack a significant 
amount of luminal lipid debris, and have resolvable lipid domains. For homogeneous GUVs, the GUVs had to be $5 \mu \mathrm{m}$ in diameter, lack a significant amount of luminal lipid debris, and lack resolvable lipid domains.

\section{Fitting the average phase separation percentage data to sigmoidal function}

The average percent phase separation data for composition 1 and composition 2 were fit using the Boltzmann sigmoidal function, $y=\frac{A_{1}-A_{2}}{1+e^{\left(x-x_{0}\right) / d x}}+A_{2}$ where $A_{1}$ is the initial value, $A_{2}$ is the final value, $x$ is the concentration of PEG lipid, $x_{0}$ is the concentration of PEG lipid at the center of the sigmoid, and $d x$ is the width of the sigmoid. $A_{1}$ is fixed as the highest average percent phase separation of each PEG length. $A_{2}$ is fixed to 0 for every PEG length.

Since $A_{2}$ is 0 the fitting equation takes this form: $y=\frac{A_{1}}{1+e^{\left(x-x_{0}\right) / d x}}$.

\section{Estimating the molar fraction of PEG-conjugated lipids required to suppress $L_{0}-L_{D}$ phase separation}

The transition point occurs when the concentration of PEG lipid is equal to the concentration of PEG lipid at the center of the sigmoidal function (where $\mathrm{x}=\mathrm{x}_{0}$ ). When $\mathrm{x}=\mathrm{x}_{0}$ the Boltzmann equation used to make the fits takes the following form: $y=\frac{A_{1}}{2}$. Therefore, the transition point is concentration of PEG-conjugated lipid where the initial average phase separation percentage is halved.

\section{Image analysis to determine partitioning factor}

The partitioning factor of fPEG2000-DSPE to the liquid ordered phase was estimated as follows. A low concentration of fPEG2000-DSPE $(0.1 \mathrm{~mol} \%)$ was used to eliminate the potential for self-quenching among the fluorescent-labeled PEG chains. A single confocal slice of the phase separated GUV sample was acquired. The image processing program ImageJ was used to analyze the images. The line profile tool was used to capture the background-subtracted peak intensity of the liquid ordered and liquid disordered domains. Then the partitioning factor was calculated by dividing the background-subtracted peak intensity of the liquid ordered domain by the background-subtracted peak intensity of the liquid disordered domain. Partitioning factors calculated from at least 39 GUVs were averaged to arrive at each data point. For GUVs labeled with both Texas Red and fPEG2000-DSPE in Figures 1 and 5, 0.3 mol\% of each label was used. However, these vesicles were used for visualization and qualitative comparisons only, not for calculation of partitioning factors.

\section{Estimating the molar concentration of PEG-conjugated lipids in the ordered phase}

By using the partitioning factor, the area fraction of the ordered phase, and the total molar concentration of PEG-conjugated lipids in the membranes, we can estimate the molar concentration of PEG-conjugated lipids in the ordered phase.

$$
C_{P E G-D}=P_{F} C_{P E G-N D} \quad \text { (Eqn. 1) }
$$


Where $C_{P E G-D}$ is the molar concentration of PEG in the domain, $C_{P E G-N D}$ is the molar concentration of PEG not in the domain, and $P_{F}$ is the partitioning factor. Next, an area balance was done.

$$
\frac{A_{D}}{A_{T O T}}+\frac{A_{N D}}{A_{T O T}}=1 \quad \text { (Eqn. 2) }
$$

Where $A_{D}$ is the surface area of the domain, $A_{N D}$ is the surface area of not the domain, and $A_{T O T}$ is the total surface area of the vesicle. The ratio of $\frac{A_{D}}{A_{T O T}}$ was determined earlier by measuring the vesicle dimensions in imageJ. By using conservation of mass we can then determine the concentration of PEG in the domain.

$C_{P E G-D}\left(\frac{A_{D}}{A_{T O T}}\right)+C_{P E G-N D}\left(\frac{A_{N D}}{A_{T O T}}\right)=C_{P E G-T O T}$ (Refer to the assumptions below)

\section{(Eqn. 3)}

Where $C_{P E G-T O T}$ is the total molar concentration of PEG in the membrane.

Now by substituting the first two equations into the third, we have,

$$
C_{P E G-D}\left(\frac{A_{D}}{A_{T O T}}\right)+\frac{C_{P E G-D}\left(1-\frac{A_{D}}{A_{T O T}}\right)}{P_{F}}=C_{P E G-T O T} \quad \text { (Eqn. 4) }
$$

Finally rearranging this expression to solve for the concentration of PEG in the domain, we have

$$
C_{P E G-D}=\frac{C_{P E G-T O T}}{\left(\frac{A_{D}}{A_{t o t}}+\frac{\left(1-\frac{A_{D}}{A_{t o t}}\right)}{P_{F}}\right)} \quad \text { (Eqn. 5) }
$$

Assumptions underlying Equation 3:

We begin with the assumption that the total number of PEG-conjugated lipid molecules $\left(N_{P E G-T O T}\right)$ is simply equal to the sum of the number in the domain $\left(N_{P E G-D}\right)$ and the number outside the domain $\left(N_{P E G-N D}\right)$ :

$$
N_{P E G-T O T}=N_{P E G-D}+N_{P E G-N D}
$$

Then we define the concentrations of PEG molecules in these regions as follows: 


$$
\begin{gathered}
C_{P E G-T O T}=\frac{N_{P E G-T O T}}{A_{T O T}} \\
C_{P E G-D}=\frac{N_{P E G-D}}{A_{D}} \\
C_{P E G-N D}=\frac{N_{P E G-N D}}{A_{N D}}
\end{gathered}
$$

Here $A_{T O T}$ is the total surface area of the lipid vesicle, $A_{D}$ is the surface area of the domain, and $A_{N D}$ is the surface area of the region outside the domain. Substituting these definitions into the expression of conservation of lipid number, we derive equation 3 without invoking an area per lipid in within the membrane. Finally, in order to relate $C_{P E G-T O T}$ (which has units of number of lipids per surface area) to the molar fraction of PEG-conjugated lipids in our vesicles, we simply multiply $C_{P E G-T O T}$ by the overall average area per lipid in the vesicle, for which we used a value of $0.7 \mathrm{~nm}^{2}$

\section{Estimating the fractional coverage of the ordered phase by PEG chains}

To determine the coverage of the PEG chains we simply multiplied the approximate concentration (number per area) of PEG-conjugated lipids in the ordered phase by the projected area per PEG chain.

\section{Supplementary Material}

Refer to Web version on PubMed Central for supplementary material.

\section{Acknowledgments}

We thank David Busch, Wilton Snead, and Orrin Shindell (UT Austin) for discussion of the manuscript; and the NIH (GM1 12065 to Stachowiak) and NSF (DMR1352487 to Stachowiak) for research funding. We thank the dedicated teachers in the Science Department at Bastrop High School (Bastrop, TX) for enabling A. Carrillo and I. Espinoza to work in the Stachowiak laboratory at UT Austin.

\section{REFERENCES}

1. Suzuki KG, Kasai RS, Hirosawa KM, Nemoto YL, Ishibashi M, Miwa Y, Fujiwara TK, Kusumi A. Transient Gpi-Anchored Protein Homodimers Are Units for Raft Organization and Function. Nature chemical biology. 2012; 8:774-783. [PubMed: 22820419]

2. Kusumi A, Nakada C, Ritchie K, Murase K, Suzuki K, Murakoshi H, Kasai RS, Kondo J, Fujiwara T. Paradigm Shift of the Plasma Membrane Concept from the Two-Dimensional Continuum Fluid to the Partitioned Fluid: High-Speed Single-Molecule Tracking of Membrane Molecules. Annual review of biophysics and biomolecular structure. 2005; 34:351-378.

3. Cocucci E, Aguet F, Boulant S, Kirchhausen T. The First Five Seconds in the Life of a ClathrinCoated Pit. Cell. 2012; 150:495-507. [PubMed: 22863004]

4. Schmid EM, McMahon HT. Integrating Molecular and Network Biology to Decode Endocytosis. Nature. 2007; 448:883-888. [PubMed: 17713526] 
5. Mattila PK, Lappalainen P. Filopodia: Molecular Architecture and Cellular Functions. Nat Rev Mol Cell Biol. 2008; 9:446-454. [PubMed: 18464790]

6. Lingwood D, Simons K. Lipid Rafts as a Membrane-Organizing Principle. Science. 2010; 327:4650. [PubMed: 20044567]

7. Veatch SL, Keller SL. Separation of Liquid Phases in Giant Vesicles of Ternary Mixtures of Phospholipids and Cholesterol. Biophys. J. 2003; 85:3074-3083. [PubMed: 14581208]

8. Garcia-Saez AJ, Schwille P. Stability of Lipid Domains. FEBS letters. 2010; 584:1653-1658. [PubMed: 20036662]

9. Honerkamp-Smith AR, Veatch SL, Keller SL. An Introduction to Critical Points for Biophysicists; Observations of Compositional Heterogeneity in Lipid Membranes. Biochim Biophys Acta. 2009; 1788:53-63. [PubMed: 18930706]

10. Korlach J, Schwille P, Webb WW, Feigenson GW. Characterization of Lipid Bilayer Phases by Confocal Microscopy and Fluorescence Correlation Spectroscopy. P Natl Acad Sci USA. 1999; 96:8461-8466.

11. Almeida PFF, Pokorny A, Hinderliter A. Thermodynamics of Membrane Domains. Biochim. Biophys. Acta-Biomembr. 2005; 1720:1-13.

12. Bacia K, Schuette CG, Kahya N, Jahn R, Schwille P. Snares Prefer Liquid-Disordered over "Raft" (Liquid-Ordered) Domains When Reconstituted into Giant Unilamellar Vesicles. Journal of Biological Chemistry. 2004; 279:37951-37955. [PubMed: 15226320]

13. Jacobson K, Mouritsen OG, Anderson RGW. Lipid Rafts: At a Crossroad between Cell Biology and Physics. Nature Cell Biology. 2007; 9:7-14. [PubMed: 17199125]

14. Fittipaldi A, Ferrari A, Zoppe M, Arcangeli C, Pellegrini V, Beltram F, Giacca M. Cell Membrane Lipid Rafts Mediate Caveolar Endocytosis of Hiv-1 Tat Fusion Proteins. Journal of Biological Chemistry. 2003; 278:34141-34149. [PubMed: 12773529]

15. Nguyen DH, Hildreth JEK. Evidence for Budding of Human Immunodeficiency Virus Type 1 Selectively from Glycolipid-Enriched Membrane Lipid Rafts. J Virol. 2000; 74:3264-3272. [PubMed: 10708443]

16. Takeda M, Leser GP, Russell CJ, Lamb RA. Influenza Virus Hemagglutinin Concentrates in Lipid Raft Microdomains for Efficient Viral Fusion. P Natl Acad Sci USA. 2003; 100:14610-14617.

17. Janes PW, Ley SC, Magee AI, Kabouridis PS. The Role of Lipid Rafts in T Cell Antigen Receptor (Tcr) Signalling. Semin Immunol. 2000; 12:23-34. [PubMed: 10723795]

18. Simons K, Toomre D. Lipid Rafts and Signal Transduction. Nature reviews. Molecular cell biology. 2000; 1:31-39. [PubMed: 11413487]

19. Scheve CS, Gonzales PA, Momin N, Stachowiak JC. Steric Pressure between Membrane-Bound Proteins Opposes Lipid Phase Separation. Journal of the American Chemical Society. 2013; 135:1185-1188. [PubMed: 23321000]

20. Tantos A, Han KH, Tompa P. Intrinsic Disorder in Cell Signaling and Gene Transcription. Mol Cell Endocrinol. 2012; 348:457-465. [PubMed: 21782886]

21. Bright JN, Woolf TB, Hoh JH. Predicting Properties of Intrinsically Unstructured Proteins. Prog Biophys Mol Biol. 2001; 76:131-173. [PubMed: 11709204]

22. Tompa, P. Structure and Function of Intrinsically Disordered Proteins. Chapman \& Hall/CRC Press; 2010.

23. Bender FC, Whitbeck JC, de Leon MP, Lou H, Eisenberg RJ, Cohen GH. Specific Association of Glycoprotein B with Lipid Rafts During Herpes Simplex Virus Entry. J Virol. 2003; 77:9542_ 9552. [PubMed: 12915568]

24. Steck TL. Organization of Proteins in Human Red Blood-Cell Membrane-Review. Journal of Cell Biology. 1974; 62:1-19. [PubMed: 4600883]

25. Grakoui A, Bromley SK, Sumen C, Davis MM, Shaw AS, Allen PM, Dustin ML. The Immunological Synapse: A Molecular Machine Controlling T Cell Activation. Science. 1999; 285:221-227. [PubMed: 10398592]

26. Pries AR, Secomb TW, Gaehtgens P. The Endothelial Surface Layer. Pflug Arch Eur J Phy. 2000; 440:653-666. 
27. Takamori S, Holt M, Stenius K, Lemke EA, Gronborg M, Riedel D, Urlaub H, Schenck S, Brugger B, Ringler P, Muller SA, Rammner B, Grater F, Hub JS, De Groot BL, Mieskes G, Moriyama Y, Klingauf J, Grubmuller H, Heuser J, Wieland F, Jahn R. Molecular Anatomy of a Trafficking Organelle. Cell. 2006; 127:831-846. [PubMed: 17110340]

28. Phillips, R.; Kondev, J.; Theriot, J. Physical Biology of the Cell. Garland Science; 2009.

29. Lozano MM, Longo ML. Complex Formation and Other Phase Transformations Mapped in Saturated Phosphatidylcholine/Dspe-Peg2000 Monolayers. Soft Matter. 2009; 5:1822-1834.

30. Kenworthy AK, Hristova K, Needham D, McIntosh TJ. Range and Magnitude of the Steric Pressure between Bilayers Containing Phospholipids with Covalently Attached Poly(Ethylene Glycol). Biophys. J. 1995; 68:1921-1936. [PubMed: 7612834]

31. Kenworthy AK, Simon SA, McIntosh TJ. Structure and Phase-Behavior of Lipid Suspensions Containing Phospholipids with Covalently Attached Poly(Ethylene Glycol). Biophys. J. 1995; 68:1903-1920. [PubMed: 7612833]

32. Kaufmann S, Borisov O, Textor M, Reimhult E. Mechanical Properties of Mushroom and Brush Poly(Ethylene Glycol)-Phospholipid Membranes. Soft Matter. 2011; 7:9267-9275.

33. Lipowsky R. Bending of Membranes by Anchored Polymers. Eurphysics Letters. 1995; 30:197202.

34. Tung C, Cacciuto A. Phase Separation of Mixed Polymer Brushes on Surfaces with Nonuniform Curvature. Journal of Chemical Physics. 2013; 139

35. Bickel T, Jeppesen C, Marques CM. Local Entropic Effects of Polymers Grafted to Soft Interfaces. European Physical Journal E. 2001; 4:33-43.

36. Immordino ML, Dosio F, Cattel L. Stealth Liposomes: Review of the Basic Science, Rationale, and Clinical Applications, Existing and Potential. Int J Nanomedicine. 2006; 1:297-315. [PubMed: 17717971]

37. Minner DE, Rauch P, Kas J, Naumann CA. Polymer-Tethered Lipid Multi-Bilayers: A Biomembrane-Mimicking Cell Substrate to Probe Cellular Mechano-Sensing. Soft Matter. 2014; 10:1189-1198. [PubMed: 24652490]

38. Morigaki K, Mizutani K, Saito M, Okazaki T, Nakajima Y, Tatsu Y, Imaishi H. Surface Functionalization of a Polymeric Lipid Bilayer for Coupling a Model Biological Membrane with Molecules, Cells, and Microstructures. Langmuir. 2013; 29:2722-2730. [PubMed: 23347422]

39. Majewski J, Wong JY, Park CK, Seitz M, Israelachvili JN, Smith GS. Structural Studies of Polymer-Cushioned Lipid Bilayers. Biophysical Journal. 1998; 75:2363-2367. [PubMed: 9788931]

40. Kuhl TL, Majewski J, Howes PB, Kjaer K, von Nahmen A, Lee KYC, Ocko B, Israelachvili JN, Smith GS. Packing Stress Relaxation in Polymer-Lipid Monolayers at the Air-Water Interface: An X-Ray Grazing-Incidence Diffraction and Reflectivity Study. Journal of the American Chemical Society. 1999; 121:7682-7688.

41. Baumgart T, Hunt G, Farkas ER, Webb WW, Feigenson GW. Fluorescence Probe Partitioning between L-O/L-D Phases in Lipid Membranes. Biochim. Biophys. Acta-Biomembr. 2007; 1768:2182-2194.

42. Skaug MJ, Longo ML, Faller R. The Impact of Texas Red on Lipid Bilayer Properties. J Phys Chem B. 2011; 115:8500-8505. [PubMed: 21644587]

43. Veatch SL, Polozov IV, Gawrisch K, Keller SL. Liquid Domains in Vesicles Investigated by Nmr and Fluorescence Microscopy. Biophys. J. 2004; 86:2910-2922. [PubMed: 15111407]

44. Carnahan NF, Starling KE. Equation of State for Nonattracting Rigid Spheres. Journal of Chemical Physics. 1969; 51:635.

45. Song Y, Mason EA, Stratt RM. Why Does the Carnahan-Starling Equation Work So Well? Journal of Physical Chemistry. 1989; 93:6916-6919.

46. Hac AE, Seeger HM, Fidorra M, Heimburg T. Diffusion in Two-Component Lipid Membranes - a Fluorescence Correlation Spectroscopy and Monte Carlo Simulation Study. Biophys. J. 2005; 88:317-333. [PubMed: 15501937]

47. Carignano MA, Szleifer I. On the Structure and Pressure of Tethered Polymer Layers in Good Solvent. Macromolecules. 1995; 28:3197-3204. 
48. Tkachenko E, Simons M. Clustering Induces Redistribution of Syndecan-4 Core Protein into Raft Membrane Domains. Journal of Biological Chemistry. 2002; 277:19946-19951. [PubMed: 11889131]

49. Angelova M, Dimitrov D. Liposome Electroformation. Faraday Discussions. 1986; 81:303-311.

50. Blosser MC, Starr JB, Turtle CW, Ashcraft J, Keller SL. Minimal Effect of Lipid Charge on Membrane Miscibility Phase Behavior in Three Ternary Systems. Biophysical Journal. 2013; 104:2629-2638. [PubMed: 23790371] 

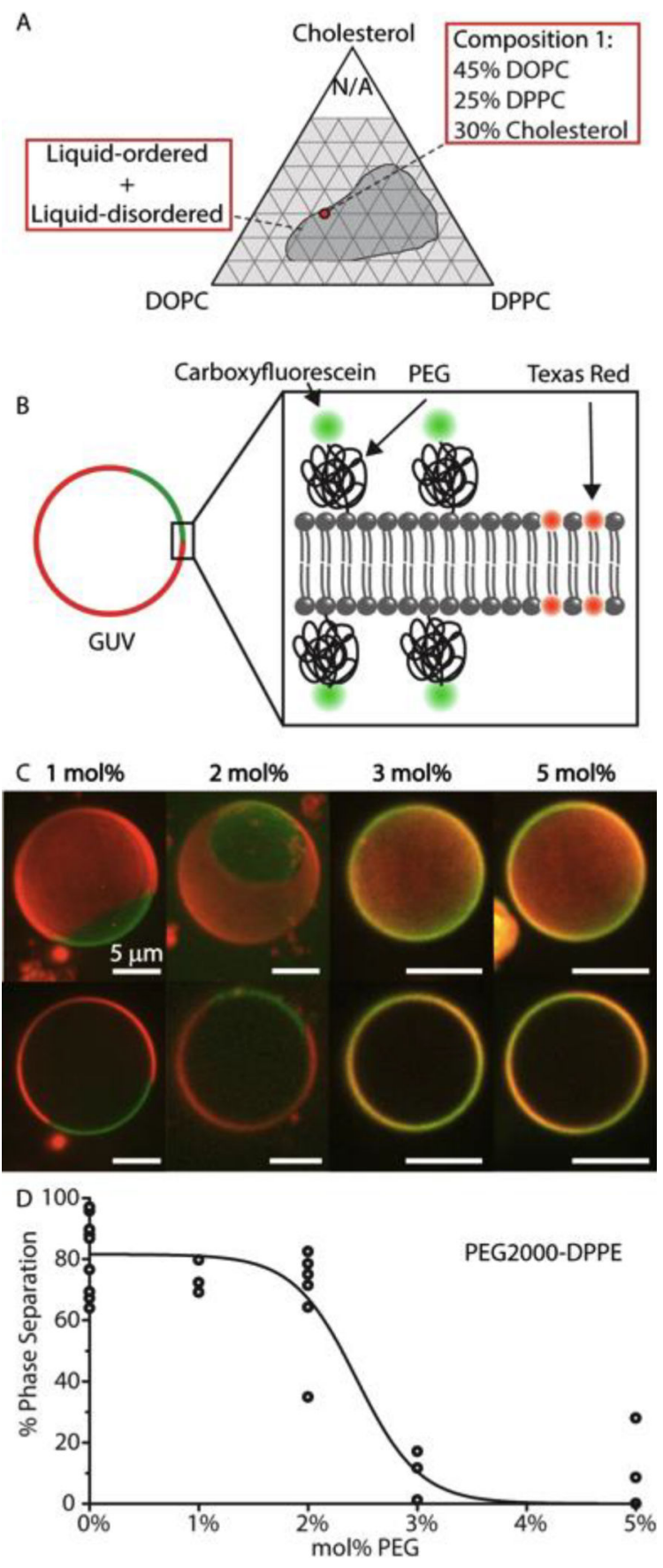

Figure 1.

Increasing the molar fraction PEG2000-DPPE destabilizes lipid domains. (A) Composition 1 consists of 45 mole percent (mol\%) DOPC, $30 \mathrm{~mol} \%$ Cholesterol, and $25 \mathrm{~mol} \%$ DPPC. This composition corresponds to the point shown in lipid phase diagram shown in Figure 1A. This phase diagram depicts the range of composition where liquid-liquid coexistence can occur at $25^{\circ} \mathrm{C}^{7}$ (B) The GUVs were labeled with 2 dyes, Texas Red-DPPE $(0.3 \mathrm{~mol} \%)$ and fPEG2000-DSPE (0.3 mol\%). (C) Confocal 3D reconstruction (top) and single confocal slice through the center (bottom) of GUVs containing $1 \mathrm{~mol} \%, 2 \mathrm{~mol} \%, 3 \mathrm{~mol} \%$, and $5 \mathrm{~mol}$ 
$\%$ PEG2000-DPPE (from left to right). (D) The percentage of phase separated vesicles within the GUV population as a function of increasing molar concentration of PEG2000DPPE. Each data point represents a single trial, with at least three trials per condition. The averages of the phase separation percentage data were fit to a sigmoidal Boltzmann function. The residuals of this fit are plotted in Figure S3. All scale bars correspond to $5 \mu \mathrm{m}$. 


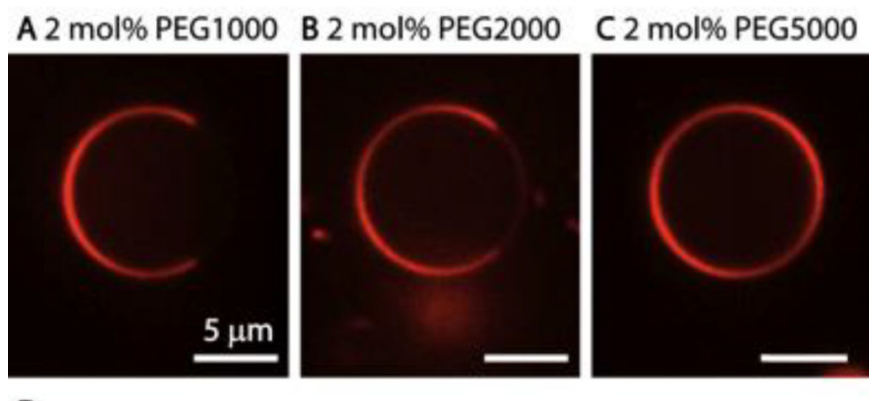

D
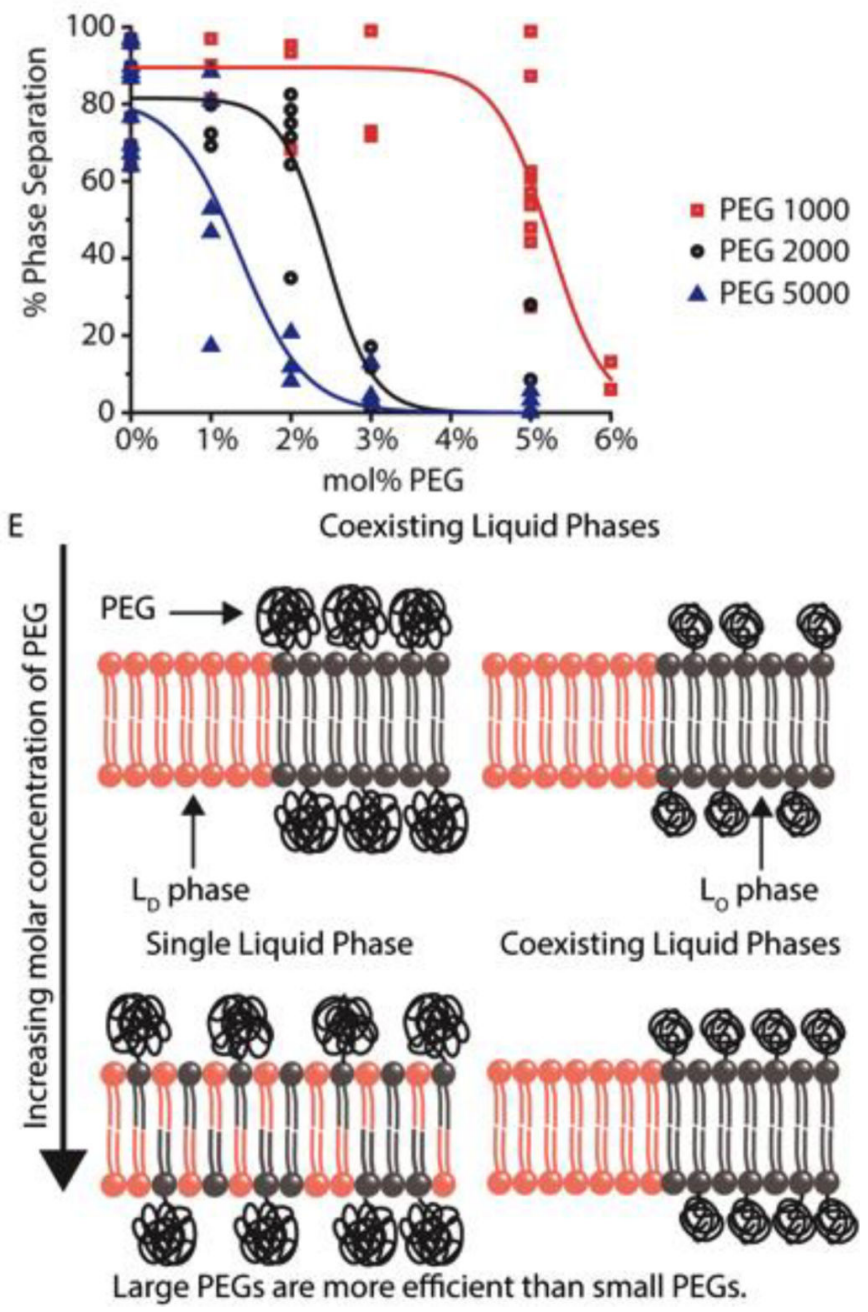

Figure 2.

As the size of PEG chains increases, the molar fraction of PEG-conjugated lipids required to suppress membrane phase separation decreases. (A-C) Fluorescence confocal image slices (0.3 mol\% Texas Red-DPPE) of GUVs containing 2 mol\% of PEG-DPPE. The GUV populations are predominantly phase separated for (A) 2 mol\% PEG1000-DPPE and (B) 2 mol\% PEG2000-DPPE, but are majority homogeneous for (C) 2 mol\% PEG5000-DPPE. (D) The percentage of phase separated vesicles within the GUV population as a function of increasing molar concentration of each of the PEG-conjugated lipids. The molar 
concentration of PEG-conjugated lipids required for lipid mixing decreases with increasing PEG chain length. Each data point represents a single trial, with at least three trials per condition. The averages of the phase separation percentage data for each PEG size were fit to a sigmoidal Boltzmann function. The residuals of these fits are plotted in Figure S3. (AD) The composition of the GUVs used in these experiments was $45 \mathrm{~mol} \%$ DOPC, $30 \mathrm{~mol} \%$ Cholesterol, and 25 mol\% DPPC/PEG-DPPE. (E) Pictorial representation of lipid mixing caused by steric pressure among membrane-conjugated PEG chains. Comparing membranes with an equal molar fraction of PEG-conjugated lipids, longer PEG chains cover a larger fraction of the membrane surface in comparison to shorter chains. When the energetic barrier to lipid mixing is relatively low, this enhanced membrane coverage gives longer PEG chains the ability to suppress membrane phase separation at lower molar fractions in comparison to shorter PEG chains. All scale bars correspond to $5 \mu \mathrm{m}$. 


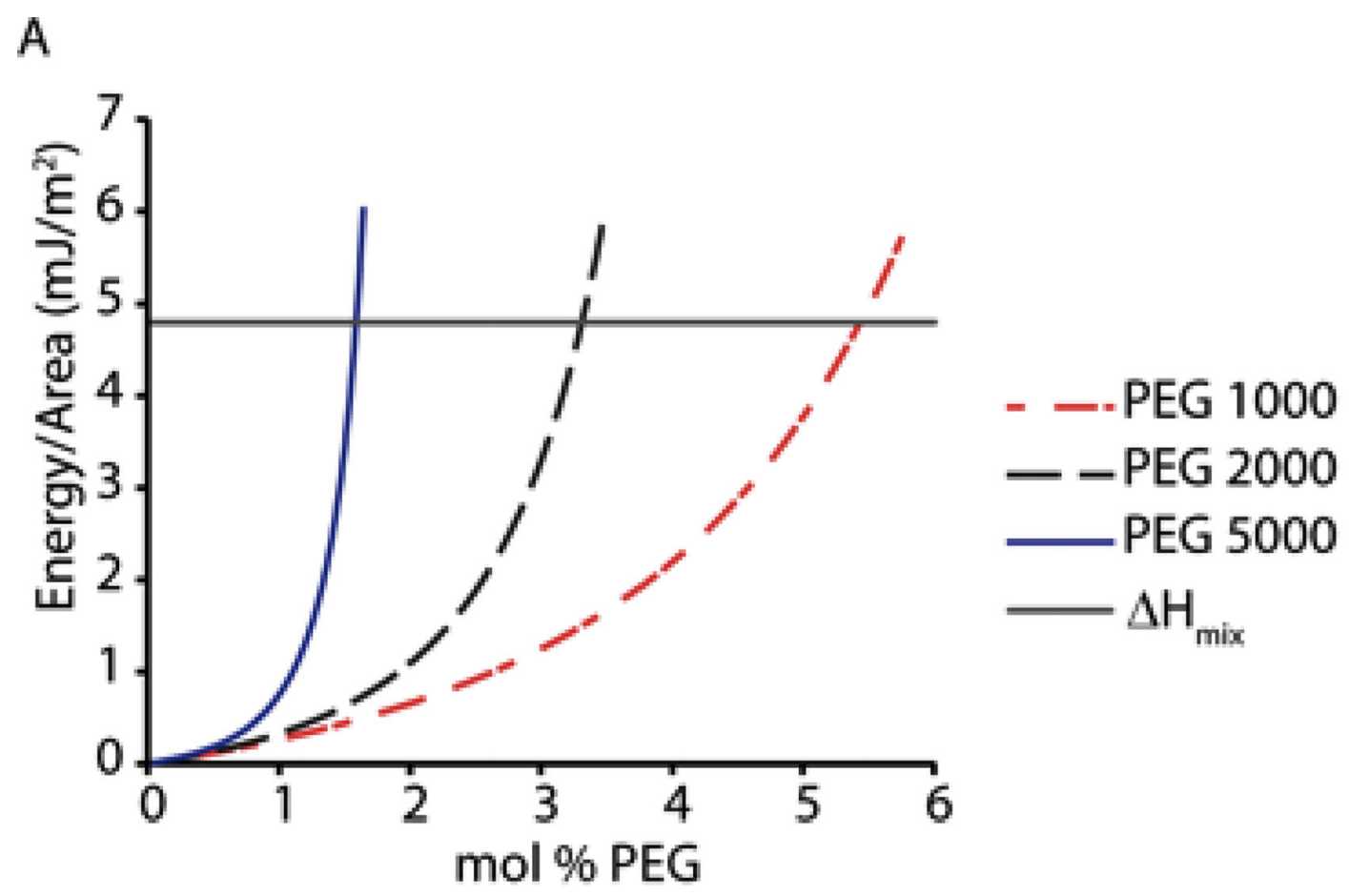

B

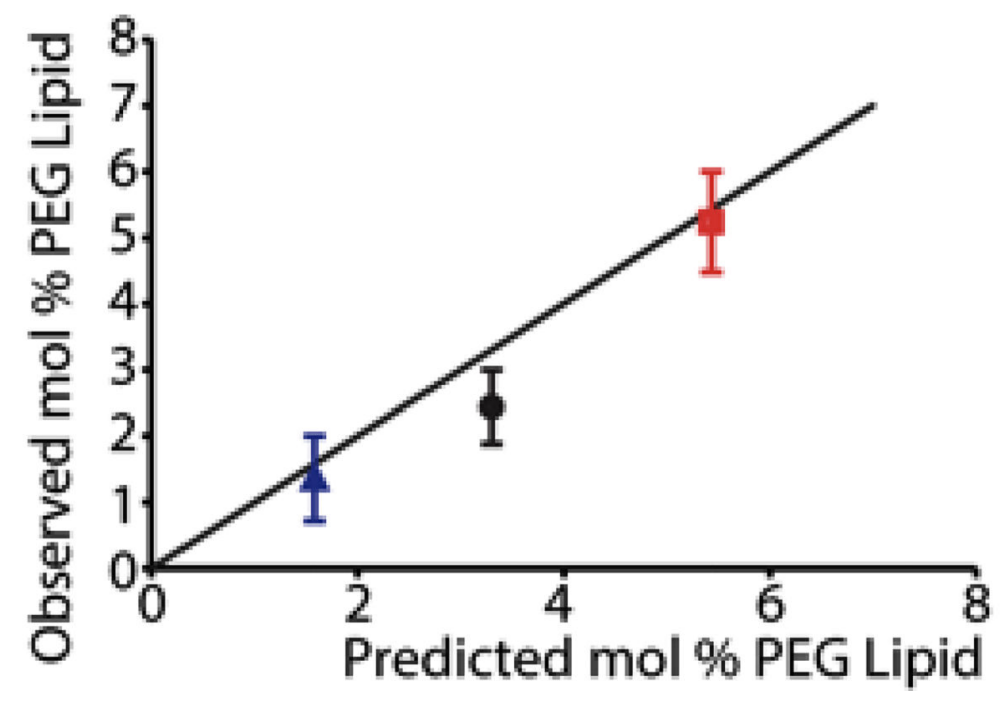

- PEG 1000

- PEG 2000

^ PEG 5000

Figure 3.

A simple steric pressure model predicts the concentrations of PEG-conjugated lipids required to destabilize membrane phase separation. (A) Predicted 2D steric pressure $\left(\frac{m J}{m^{2}}\right)$ as a function of the concentration of PEG-conjugated lipids in the liquid ordered phase. The steric pressure generated by the PEG chains increases until it surpasses the enthalpy of mixing (horizontal line). (B) Comparison of the experimentally observed and predicted concentrations of PEG-conjugated lipids required to destabilize lipid domains. Experimental 
data are from Figure 2D. The line represents a 1:1 agreement between predicted mol\% PEG lipid and the observed mol \% PEG lipid. The error bars represent the range of concentrations over which the major transition in Figure 2D takes place. 
A
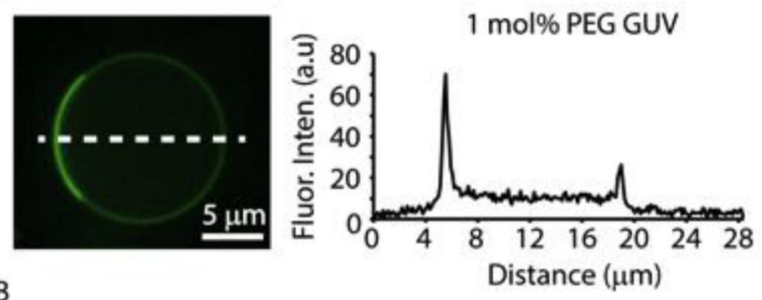

B

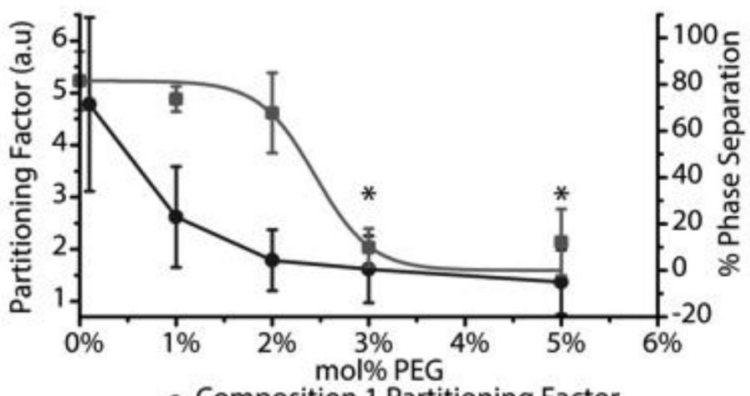

- Composition 1 Partitioning Factor

- Composition 1 \% Phase Separation

C Cholesterol

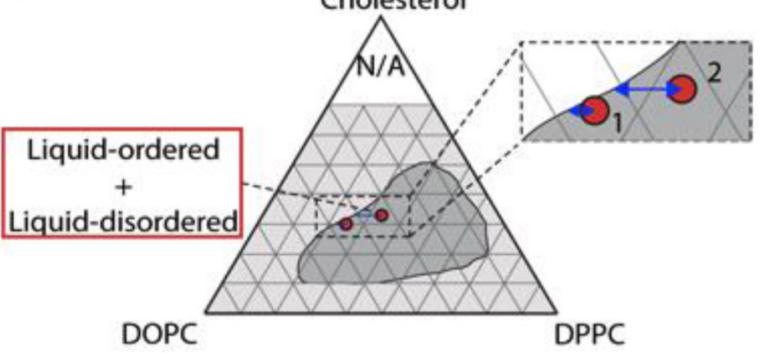

D

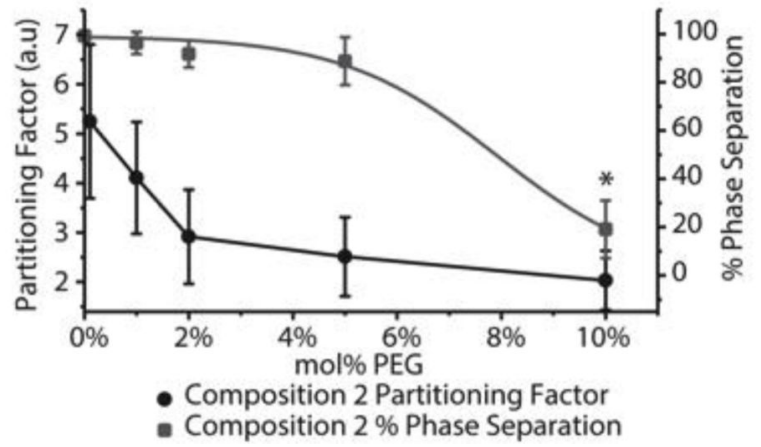

Figure 4.

Steric pressure among PEG chains decreases the preference of PEG-conjugated lipids for the liquid ordered membrane phase. (A) A single confocal slice through the center of a composition 1 phase separated vesicle containing 1 mol\% PEG2000-DPPE labeled with 0.1 mol\% fPEG2000-DSPE and corresponding line profile. (B) Combined plots showing the fraction of phase-separated vesicles (right axis) and the partitioning factor of fPEG2000DSPE to the ordered phase (left axis), both versus the molar fraction of PEG2000-DPPE for vesicles of composition 1 . The averages of the phase separation percentage data were fit to a 
sigmoidal Boltzmann function. The residuals of this fit are plotted in Figure S3. (C) Ternary membrane phase diagram depicting the liquid-liquid coexistence region at $25^{\circ} \mathrm{C}$, based on data in the literature. ${ }^{7}$ Compositions 1 and 2 are labeled, where the relative proximity of the two compositions to the phase boundary (inset) indicates that the energetic barrier to lipid mixing is greater for composition 2 than for composition 1. (D) Combined plots showing the fraction of phase-separated vesicles (right axis) and the partitioning factor of fPEG2000DSPE to the ordered phase (left axis), both versus the molar fraction of PEG2000-DPPE for vesicles of composition 2. Each point of the partitioning factor data represents the average partitioning factor of at least 39 vesicles. Error bars represent the standard deviation. Each point of the fraction of phase separation data represents the average of 3 to 9 trials. The averages of the phase separation percentage data were fit to a sigmoidal Boltzmann function. The residuals of this fit are plotted in Figure S4. The asterisk represents mixtures for which less than half of vesicles remain phase separated, such that the partitioning factor is calculated based on a minority of the total population. All scale bars correspond to $5 \mu \mathrm{m}$. 

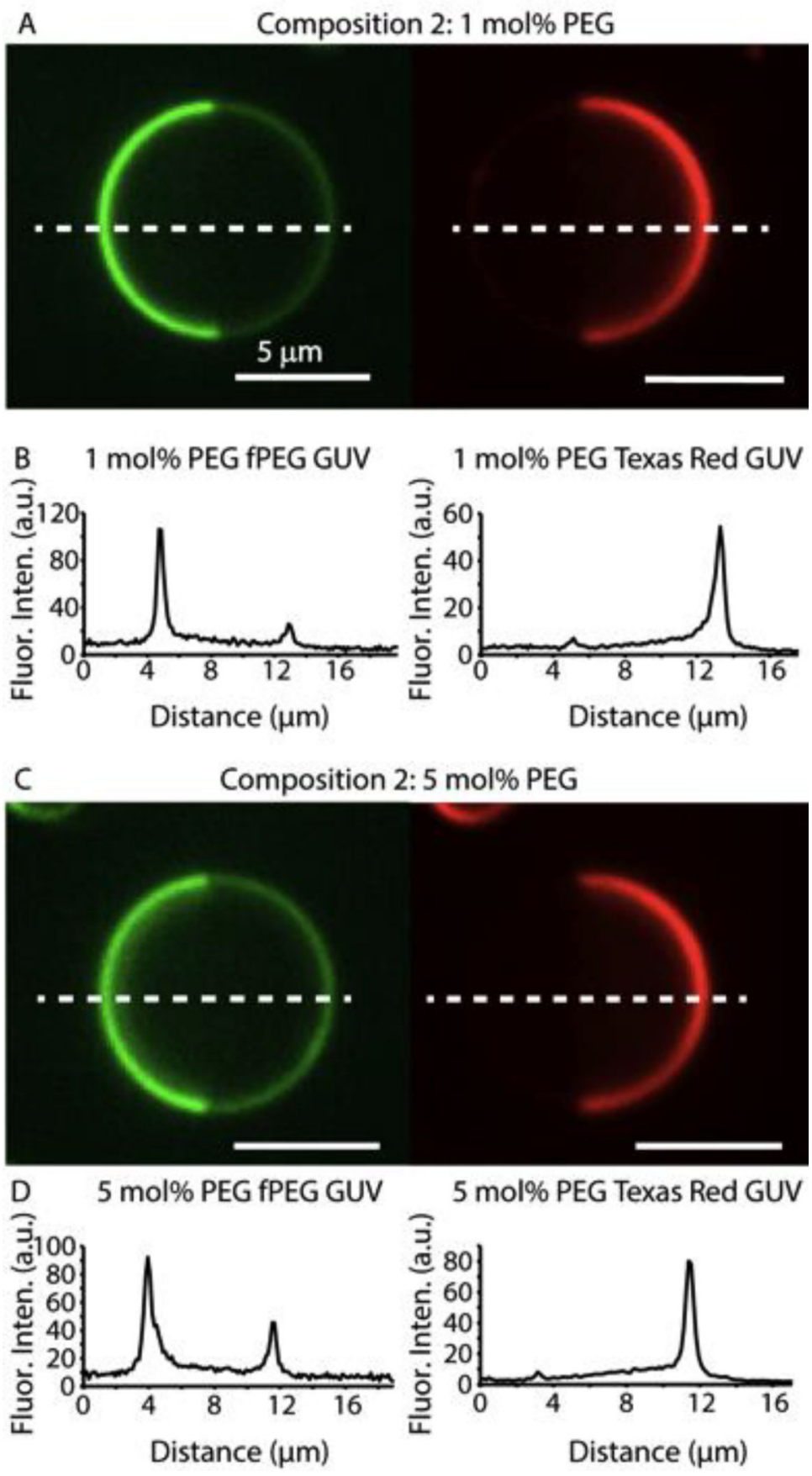

Figure 5.

As the energetic barrier to lipid mixing increases, significant repartitioning of PEGconjugated lipids to the liquid disordered phase occurs before lipid domains are destabilized. Images of GUVs of composition 2 (see Figure 4B). A single confocal slice through the center of a phase separated vesicle of composition 2, containing 1 mol\% PEG2000-DPPE (A) and 5 mol\% PEG2000-DPPE (C) labeled with 0.3 mol\% fPEG2000-DSPE (left) and 0.3 mol\% Texas Red-DPPE (right). Line profiles of the fluorescence intensity of the phase separated vesicle versus line profile length for each label fPEG2000-DSPE (left) and Texas 
Red-DPPE (right) for vesicles containing $1 \mathrm{~mol} \%$ PEG2000-DPPE (B) and 5 mol\% PEG2000-DPPE (D). The two peaks on each line profile correspond to the labeled membrane. All scale bars correspond to $5 \mu \mathrm{m}$. 

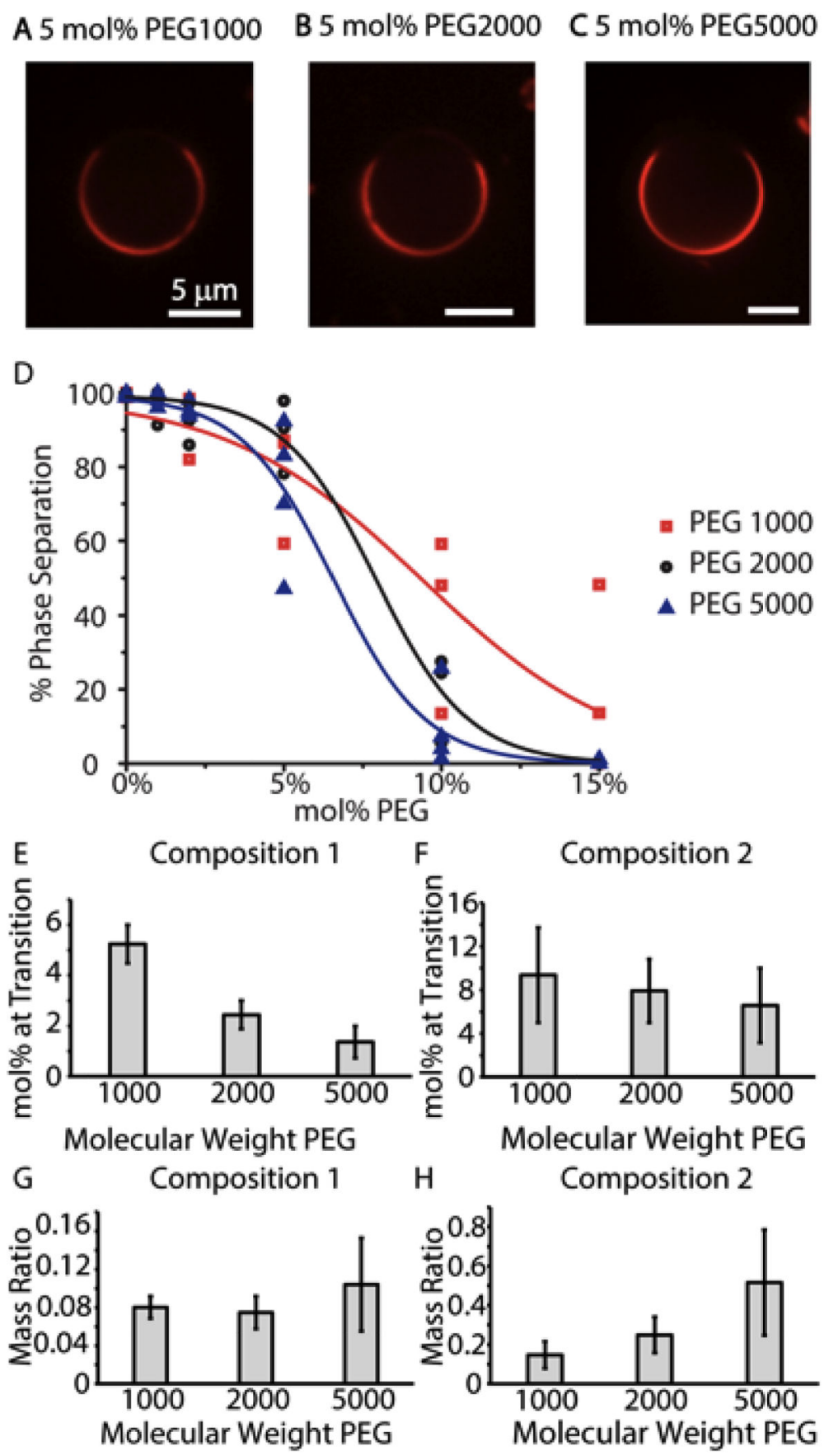

Figure 6.

Increasing the miscibility phase transition temperature increases the concentration of PEGconjugated lipids required to suppress membrane phase separation and decreases the dependency of PEG-chain length on this transition. Fluorescence confocal image slices (0.3 mol\% Texas Red-DPPE) of phase separated GUVs containing 5 mol\% of (A) PEG1000DPPE, (B) PEG2000-DPPE, and (C) PEG5000-DPPE. (D) The percentage of phase separated vesicles within the GUV population as a function of increasing molar concentration of each of the PEG-conjugated lipids. Each data point represents a single trial, 
with at least 3 trials for each experimental condition. The averages of the phase separation percentage data for each PEG size were fit to a sigmoidal Boltzmann function. The residuals of these fits are plotted in Figure S4. (A-D) The composition of the GUVs used in these experiments was $33 \mathrm{~mol} \%$ DOPC, $33 \mathrm{~mol} \%$ cholesterol and $34 \mathrm{~mol} \%$ DPPC/PEG-DPPE. (E) Bar chart showing the molar fraction of PEG-conjugated lipids at the observed transition points as a function of PEG molecular weight. Error bars represent the range of concentrations over which the transition in Figure 2D takes place. (F) Bar chart showing the molar fraction of PEG-conjugated lipids at the observed transition points as a function of PEG molecular weight. Error bars represent the range of concentrations over which the transition in Figure 6D takes place. (G) Bar chart showing the PEG to lipid mass ratio at the observed transition points as a function of PEG molecular weight. Error bars represent the range of PEG to lipid mass ratios at the observed transition points. $(\mathrm{H})$ Bar chart showing the PEG to lipid mass ratio at the observed transition points as a function of PEG molecular weight. Error bars represent the range of PEG to lipid mass ratios at the observed transition points. All scale bars correspond to $5 \mu \mathrm{m}$. 
A

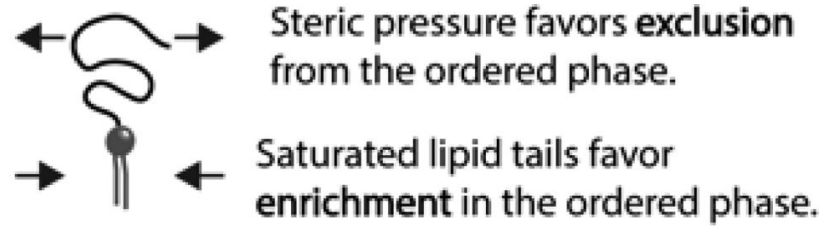

B Coexisting Liquid Phases

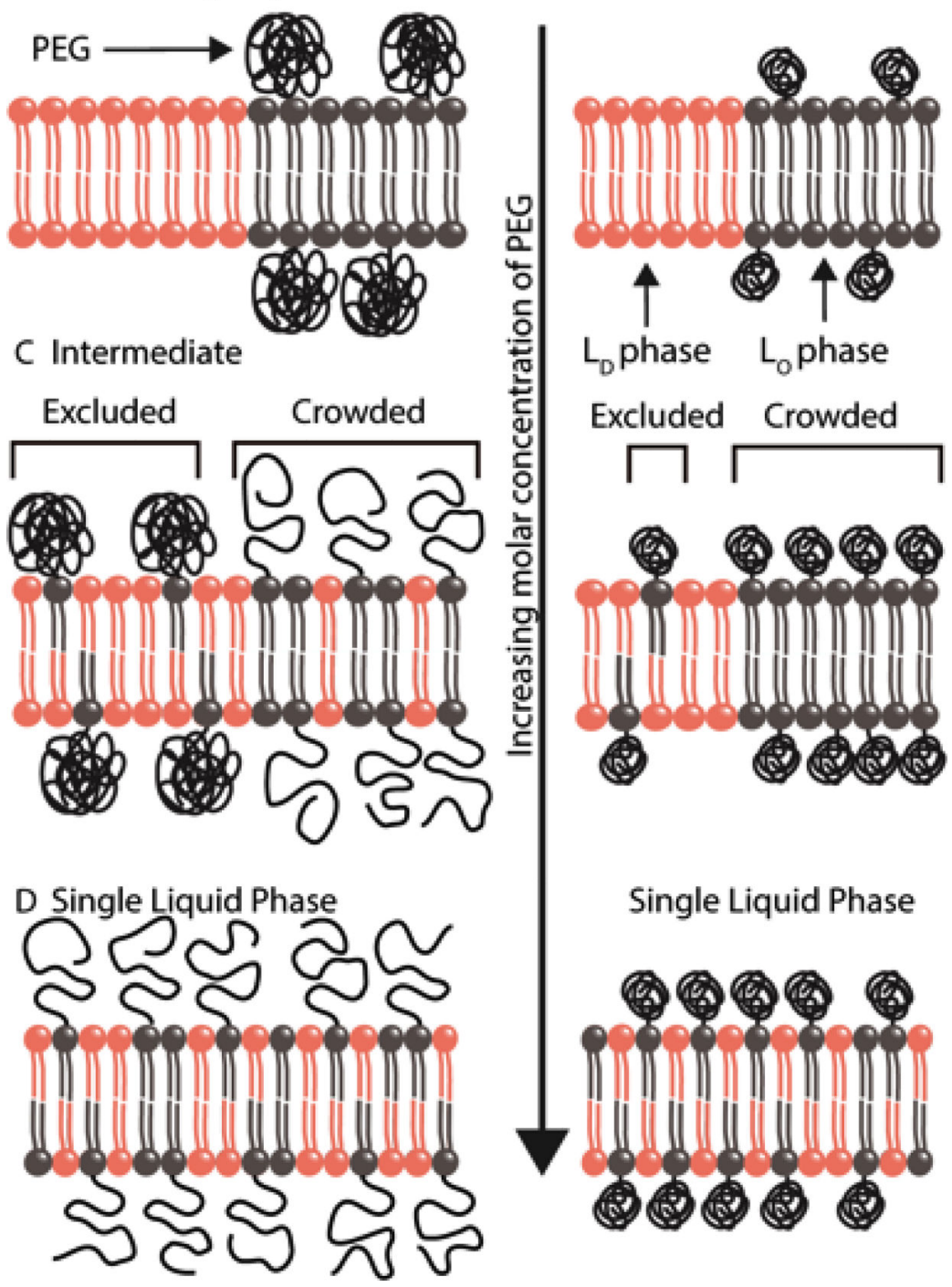

Figure 7.

As the energetic barrier to lipid mixing increases, steric repartitioning of polymerconjugated lipids to the disordered phase reduces the ability of polymer-conjugated lipids to generate steric pressure in the ordered phase. (A) Energetic balance on individual PEGconjugated lipids. (B) Comparing membranes with an equal mole fraction of PEGconjugated lipids, longer PEG chains cover a larger fraction of the membrane surface in comparison to shorter chains, such that steric pressure is larger among the longer chains. (C) When the energetic barrier to lipid mixing is relatively high, the steric pressure required to 
suppress membrane phase separation increases, requiring more PEG-conjugated lipids to suppress membrane phase separation. Increasing the PEG-conjugated lipid concentration will increase polymer crowding in the $\mathrm{L}_{\mathrm{O}}$ phase, leading to steric exclusion of PEGconjugated lipids (repartitioning) to the $\mathrm{L}_{\mathrm{D}}$ phase. At equal steric pressure, longer PEG chains will repartition more readily than smaller PEG chains, as discussed in the text. (D) The disproportionate impact of repartitioning and straightening on larger PEG chains diminishes their advantage in ability to crowd the $\mathrm{L}_{O}$ phase (B). It is important to note that despite steric repartitioning of the polymer-conjugated lipid to the $\mathrm{L}_{\mathrm{D}}$ phase, the $\mathrm{L}_{\mathrm{O}}$ phase will be always more crowded than the $\mathrm{L}_{\mathrm{D}}$ phase owing to the tail-based preference of the PEG-conjugated lipids for the $\mathrm{L}_{\mathrm{O}}$ phase. 